\title{
ANÁLISE DA DEMANDA DE MEDICAMENTOS PARA USO OFF LABEL POR MEIO DE AÇÕES JUDICIAIS NA SECRETARIA DE ESTADO DE SAÚDE DE MINAS GERAIS
}

\author{
Analysis of the demand for medicines for off label use through \\ lawsuits in the State Health Secretariat of Minas Gerais
}

\author{
Karina Alves Ramos* \\ Anísia da Soledade Dias Ferreira**
}

\begin{abstract}
RESUMO
Este trabalho objetivou investigar a ocorrência de pedido judicial de medicamento para indicações não aprovadas pela Agência Nacional de Vigilância Sanitária, por meio de ações judiciais contra o Estado de Minas Gerais no ano de 2010. É um estudo transversal, exploratório, com coleta retrospectiva de dados primários. A fonte dos dados foi o parecer técnico referente à solicitação de medicamento realizada por ordem judicial. Os dados considerados foram: patologia informada na ação judicial, medicamento solicitado e a concessão, ou não, de antecipação de tutela ou de liminar. No total de 1.981 pareceres analisados, foram identificadas 212 solicitações de medicamentos para uso off label. Dentre esses medicamentos, $46 \%$ não estavam incluídos em programas de assistência farmacêutica do Sistema Único de Saúde. Os medicamentos mais solicitados para uso off label foram micofenolato de mofetila (23\%), bevacizumabe $(11 \%)$ e rituximabe $(8 \%)$. A classe terapêutica mais solicitada, de acordo com Sistema Anatômico e Terapêutico Químico, foi a dos agentes

$\left(^{\star}\right)$ Especialista em Direito Sanitário, Escola de Saúde Pública do Estado de Minas Gerais (ESP/ MG); Especialista em Vigilância Sanitária, Pontifícia Universidade Católica de Goiás (PUC/GO). Farmacêutica na Secretaria de Estado de Saúde de Minas Gerais. Belo Horizonte/MG - Brasil. E-mail: karinaaramos@yahoo.com.br.

$\left.{ }^{(*}\right)$ Mestre em Epidemiologia, Escola de Veterinária, Universidade Federal de Minas Gerais (UFMG); Especialista em Auditoria de Serviços de Saúde, Escola Nacional de Saúde Pública Sérgio Arouca (ENSP).; Especialista em Políticas e Gestão da Saúde na Secretaria de Estado de Saúde de Minas Gerais; Pesquisadora e Coordenadora de Campo do Grupo Interdisciplinar de Pesquisa em HTLV da Fundação Hemominas. Belo Horizonte/MG - Brasil. E-mail: anisia.soledade@gmail.com.
\end{abstract}

Artigo recebido em: 06/02/2012. Revisado em: 01/11/2012. Aprovado em: 07/11/2012. 
antineoplásicos e imunomoduladores (54,20\%). Dentre os medicamentos solicitados para uso off label, 78,30\% foram obtidos por meio de antecipação de tutela ou de liminar. Os resultados apontam para a necessidade de o Poder Judiciário continuar na busca pelo entendimento das questões técnicas que norteiam essas ações judiciais e de os gestores evoluírem na busca pela melhoria da assistência farmacêutica, sem, entretanto, deixar de atender àqueles casos não previstos nos protocolos clínicos, mas que, pelo avanço da ciência e por suas peculiaridades, se mostrarem necessários.

Palavras-chave: Ações Judiciais; Anvisa; Off Label; Medicamento.

\section{ABSTRACT}

This study aimed at investigating the occurrence of medication use for indications not approved by the Brazilian National Health Surveillance Agency through lawsuits against the State of Minas Gerais in 2010. The study is classified as cross-sectional exploratory retrospective collection of primary data. The data source of this research was the technical report, referring to the request of medicine held by court order. We considered the following data: disease reported in the lawsuits, requested medication and the granting or not the medicine requested through application for legal protection, or injunction. In the total of 1981 technical reports analyzed, we identified 212 requests for use of drugs off label. Among these drugs, $46 \%$ were not included in programs of pharmaceutical care of the Brazilian Public Health System. The drugs most requested for off label use were mycophenolate mofetil (23\%), bevacizumab (11\%) and rituximab $(8 \%)$. The most requested therapeutic class, according to the Anatomical and Chemical Therapeutic System, was antineoplastic and immunomodulating agents $(54.20 \%) .78 .30 \%$ of the drugs required for off label use were obtained by means of legal protection, or injunction. The results point to the need for the judiciary to continue in the search for understanding of the technical issues that permeate these lawsuits and to the need for the public managers to evolve in the pursuit of improving the pharmaceutical care, without, however, fail to meet the cases not provided in the protocols clinical, but by the advancement of science and by its peculiarities, appear necessary.

Keywords: Brazilian National Health Surveillance Agency; Drugs; Lawsuits; Off Label.

\section{Introdução}

A Constituição Federal de 1988 estabelece, entre os direitos sociais, o direito à saúde, orientado pelos princípios da universalidade, integralidade e equidade, mediante a garantia de acesso do cidadão às ações e aos serviços para promoção, proteção e recuperação da saúde. Assim, o direito à saúde, como 
direito social que é, exige uma prestação positiva do Estado, a fim de garantir a sua efetivação.

Além de se qualificar como um direito social que assiste a todas as pessoas, o direito à saúde representa consequência indissociável do direito à vida. Sendo assim, o Estado não pode se mostrar omisso diante de um problema de saúde de um cidadão, sob pena de incidir em grave comportamento inconstitucional e poder ser demandado judicialmente para garantir, entre outras ações, a assistência terapêutica integral, inclusive farmacêutica.

Em decorrência disso, no Brasil pós-1988, o fornecimento de medicamentos por meio de ações judiciais, fenômeno também conhecido como "judicialização da saúde", vem crescendo e obrigando os gestores públicos a buscar o difícil equilíbrio entre demandas individuais e coletivas. Em Minas Gerais, somente no ano de 2011, foram gastos mais de $\mathrm{R} \$ 86$ milhões com o fornecimento de medicamentos por meio de ações judiciais. ${ }^{(1)}$

Além do aspecto da legitimidade do atendimento de demandas individuais, que podem impactar financeiramente na assistência à população usuária do Sistema Único de Saúde (SUS), outros aspectos devem ser observados, entre eles a segurança do usuário do medicamento demandado judicialmente. Existem características específicas desse tipo de demanda que exigem uma atenção especial do Poder Judiciário na ocasião de análise dos processos, como no caso de prescrições de medicamentos "novos" ou de "novas" indicações terapêuticas para os quais as evidências científicas ainda não se encontram bem estabelecidas e cujo fornecimento não está previsto em protocolos clínicos do SUS.

Nessa perspectiva, cabe ressaltar a expressiva quantidade de solicitação pela via judicial, que tem sido mencionada em alguns estudos, de medicamentos para uso em indicações não previstas na bula de produtos registrada junto ao órgão sanitário, caracterizando uso na forma off label.

O uso off label de medicamentos, embora possa ser apropriado em muitos casos, como em pacientes com doença grave para a qual não existem alternativas terapêuticas, pode representar sérios riscos à saúde do individuo porque, muitas vezes, quando não existe aprovação de uso de um medicamento para determinadas indicações é porque faltam informações sobre sua segurança e eficácia.

Diante desse contexto, este trabalho objetivou investigar a ocorrência de uso de medicamento para indicações não aprovadas pela Agência Nacional de Vigilância

\footnotetext{
1 Dados obtidos no PORTAL da Transparência do Estado de Minas Gerais. Disponível em: <http://www.transparencia.mg.gov.br/despesas-do-estado?u=cHVibGljYWRvci5waHA ZGF0YT1ZV05oYjN4d2NtOTRhVzF2ZkhSbGJXRjhOSHhqYjJScFoyOThOREV3TkRJNU1UY3d NalF 6 TUh 4 MFpXMXdiM0poYkdsa1IXUmxmRUZPVIVGTWZIVnVhV1JoWkd WOE1YeGhibTk4TWpBeE1YeHVhWFpsYkh3MmZIUmxiR0Y4ZkhCaGFYdzBNVEEwTWpreE56 QXIOQT09>. Acesso em: 11 jan. 2012.
} 
Sanitária (Anvisa) por meio de ações judiciais contra o Estado de Minas Gerais no ano de 2010, tendo em vista aspectos relacionados à segurança e à eficácia dos medicamentos dispensados sob ordem judicial.

\section{O Uso off label de medicamentos}

No Brasil, o registro de medicamentos é ato privativo do órgão competente do Ministério da Saúde, a Anvisa. Dentre as competências desse órgão, conforme se depreende da Lei $n^{\circ} 9.782 / 99,{ }^{(2)}$ está a de regulamentar, controlar e fiscalizar os produtos e serviços que envolvam risco à saúde pública, entre os quais medicamentos de uso humano, suas substâncias ativas e demais insumos, processos e tecnologias.

Cada medicamento registrado no Brasil recebe a aprovação da Anvisa para uma ou mais indicações, as quais precisam ser comprovadas por meio da apresentação de estudos clínicos robustos e confiáveis. A partir de sua aprovação pelo órgão sanitário, a indicação do medicamento passa a constar em sua bula; sua utilização, para essa finalidade, é respaldada pela Agência.

Segundo a Resolução RDC n 47/09,(3) a bula é o documento legal sanitário que contém informações técnico-científicas orientadoras sobre os medicamentos para o seu uso racional. Assim, todo uso de um medicamento em situações divergentes das que constam na bula do medicamento registrado junto à Anvisa é considerado off label, não sendo aprovado pelo órgão sanitário.

Segundo Gazarian et al.,(4) prescrições off label se referem àquelas prescrições de medicamentos para um uso que não está incluído nas informações do produto (em bula). Exemplos incluem a prescrição do uso de um medicamento para uma indicação, faixa etária, dose, ou ainda, via de administração não recomendadas em bula.

A utilização de um medicamento já registrado para uma indicação não regulamentada é uma prática bastante difundida. Em 2001, estimaram-se 150 milhões de usos off label de medicamentos prescritos a pacientes ambulatoriais nos

\footnotetext{
2 BRASIL. Lei $n^{\circ}$ 9.782, de 26 de janeiro de 1999. "Define o Sistema Nacional de Vigilância Sanitária e dá outras providências". Diário Oficial [da] Republica Federativa do Brasil, Brasília, DF, 27 jan. 1999. Disponível em <http://www.planalto.gov.br/ccivil_03/Leis/L9782.htm>. Acesso em: 28 out. 2010.

${ }^{3}$ BRASIL. Resolução RDC n 47 de 08 de setembro de 2009. "Estabelece regras para elaboração, harmonização, atualização, publicação e disponibilização de bulas de medicamentos para pacientes e para profissionais de saúde". Diário Oficial [da] Republica Federativa do Brasil, Brasília, DF, 09 set. 2009. Disponível em: <http://portal2.saude.gov.br/saudelegis/leg_norma_pesq_consulta.cfm> Acesso em: 23 nov. 2010.

${ }^{4}$ GAZARIAN, M.; KELLY, M.; MCPHEE, J.R.; GRAUDINS, L.V.; WARD, R.L.; CAMPBELL, T.J. Offlabel use of medicines: consensus recommendations for evaluating appropriateness. The Medical Journal of Australia, Austrália, v. 185, n.10, p. 544-547, Nov. 2006.
} 
Estados Unidos. A maioria das indicações (73\%) apresentava pouco ou nenhum suporte científico. ${ }^{(5)}$

A promoção de medicamentos para uso off label no Brasil é proibida, conforme a Resolução RDC $n^{\circ} 96 / 08$, Art. $3^{\circ}$, parágrafo $2^{\circ}$, segundo a qual:

Todas as alegações presentes na peça publicitária referentes à ação do medicamento, indicações, posologia, modo de usar, reações adversas, eficácia, segurança, qualidade e demais características do medicamento devem ser compatíveis com as informações registradas na Anvisa. ${ }^{(6)}$

É importante salientar que, embora esse tipo de propaganda seja proibido, o uso off label de medicamentos não é ilegal e, muitas vezes, pode ser clinicamente apropriado, por exemplo, no caso de um paciente com uma doença séria, para a qual não existem alternativas, e no caso em que os potenciais benefícios superam os riscos. Todavia, tal uso traz consigo uma série de questões clínicas, de segurança e éticas, as quais devem ser atentamente observadas no momento da prescrição, que deve de feita do modo mais seguro possível e com fundamento em evidências científicas confiáveis. Esse tipo de prescrição implica, portanto, grande responsabilidade do profissional, que deve avaliar os riscos e benefícios para cada paciente. ${ }^{(7)}$

Vale lembrar que o uso de um medicamento com pouca ou nenhuma evidência de eficácia e segurança e em dose não apropriada pode expor o paciente a terapias não efetivas e a riscos desconhecidos de eventos adversos. ${ }^{(8)}$

Dessa forma, o processo de tomada de decisão quanto à utilização, ou não, de um medicamento na forma off label não é algo simples e requer cuidados adicionais, como a busca exaustiva na literatura, para melhor esclarecimento sobre os potenciais benefícios e riscos envolvidos. Isso aponta para a necessidade de grande cautela por parte do profissional prescritor ao optar por essa forma de tratamento, devendo estar fundamentado nas melhores evidências científicas disponíveis.

\footnotetext{
${ }^{5}$ WANNMACHER, L. A ética do medicamento: múltiplos cenários. Ministério da Saúde. Uso racional de medicamentos: temas selecionados. Brasília, v. 4, n.8, jul. 2007.

${ }^{6}$ BRASIL. Resolução RDC $n^{\circ}$ 96, de 17 de dezembro de 2008. "Dispõe sobre a propaganda, publicidade, informação e outras práticas cujo objetivo seja a divulgação ou promoção comercial de medicamentos". Diário Oficial [da] Republica Federativa do Brasil, Brasília, DF, 18 dez. 2008. Disponível em: <http://portal2.saude.gov.br/saudelegis/leg_norma_pesq_consulta.cfm>. Acesso em: 23 nov. 2010.

${ }^{7}$ GAZARIAN, M.; KELLY, M.; MCPHEE, J.R.; GRAUDINS, L.V.; WARD, R.L.; CAMPBELL, T.J. op. cit.

${ }^{8}$ GAZARIAN, M. Off label use of medicines in the paediatric population: recommendations for assessing appropriateness. Sydney 7 May 2007. 25 p. Disponível em: <http://archives.who.int/eml/ expcom/children/Items/WHOofflabel pdf>. Acesso em: 22 out. 2012.
} 


\section{O direito constitucional à saúde e a demanda judicial por medicamentos: um enfoque sanitário}

A atuação do Poder Judiciário brasileiro na proteção dos direitos individuais e coletivos e no controle das políticas públicas, especialmente na área da saúde, vem se tornando mais frequente desde o fim da década de 1980, quando o texto constitucional de 1988 inovou ao positivar o direito à saúde, definindo as ações e serviços de saúde como de relevância pública e determinando, assim, a essencialidade de sua prestação para o interesse social.

O Art. 196 da Constituição Federal estabelece expressamente que:

A saúde é direito de todos e dever do Estado, garantido mediante políticas sociais e econômicas que visem à redução do risco de doença e de outros agravos e ao acesso universal e igualitário às ações e serviços para sua promoção, proteção e recuperação. ${ }^{(9)}$

Para garantir esse direito, por meio da Constituição Federal de 1988, foi instituída uma estrutura política complexa e abrangente para o sistema de saúde brasileiro, com a organização do SUS.

Destaca-se que o direito à assistência farmacêutica, como parte integrante do direito social à saúde, também foi inserido no ordenamento jurídico como um direito social. De acordo com os artigos $6^{\circ}$ e $7^{\circ}$ da Lei $n^{\circ} 8.080 / 90,{ }^{(10)}$ as assistências terapêuticas e farmacêuticas devem ser garantidas integralmente aos cidadãos brasileiros, de acordo com o princípio da integralidade de assistência. ${ }^{(11)}$

Segundo Aith $^{\left({ }^{12)}\right.}$ a diretriz de integralidade das ações e serviços públicos de saúde representa um importante instrumento de defesa do cidadão contra eventuais omissões do Estado. Isso implica que, sempre que houver uma pessoa doente, caberá ao Estado fornecer o tratamento terapêutico para a recuperação da saúde dessa pessoa, de acordo com as possibilidades oferecidas pelo desenvolvimento científico, não importando o nível de complexidade exigido. Todos os procedimentos terapêuticos, reconhecidos pela ciência e autorizados pelas autoridades sanitárias competentes, devem ser disponibilizados para a proteção da saúde da população.

\footnotetext{
9 BRASIL. Constituição da República Federativa do Brasil de 1988. Disponível em: <http://www. planalto.gov.br/ccivil_03/constituicao/constitui\%C3\%A7ao.htm>. Acesso em: 21 ago. 2011.

${ }^{10}$ BRASIL. Lei $n^{\circ} 8.080 / 90$. "Dispõe sobre as condições para a promoção, proteção e recuperação da saúde, a organização e o funcionamento dos serviços correspondentes e dá outras providências". Disponível em: <http://www.planalto.gov.br/ccivil_03/Leis/L8080.htm>. Acesso em: 29 mar. 2013.

11 MARQUES, S.B. O princípio constitucional da integralidade de assistência à saúde e o projeto de lei n. 219/2007: interpretação e aplicabilidade pelo Poder Judiciário. Revista de Direito Sanitário, São Paulo, v. 10, n. 2, jul./out. 2009.

${ }_{12}$ AITH, F. Curso de direito sanitário: a proteção do direito à saúde no Brasil. São Paulo: Quartier Latin, 2007.
} 
Sendo assim, nos últimos anos, as ações judiciais para fornecimento de medicamentos têm sido um importante caminho para se ter acesso a medicamentos de todo tipo, desde medicamentos já fornecidos no âmbito das políticas públicas de assistência farmacêutica até medicamentos não padronizados, muitos de última geração, sem a devida comprovação de superioridade de benefício terapêutico em relação àqueles já presentes nas listas oficiais.

Diante desse contexto, os gestores do SUS vêm sustentando que o Estado possui limitações legais e orçamentárias, que restringem o fornecimento de medicamentos àqueles que constam nos protocolos clínicos e listas oficiais do SUS, a despeito das necessidades colocadas nos autos e das necessidades terapêuticas individuais. Sustentam, ainda, que atender a alguns tipos de demandas pode colocar em risco o atendimento de toda a comunidade que não acessa o Poder Judiciário para ver seu direito garantido. ${ }^{(13)}$

Em contraste, conforme constatado em pesquisa no Estado de São Paulo, o Poder Judiciário vinha fundamentando suas decisões no argumento de que o direito de todos os indivíduos à saúde representaria uma consequência indissociável do direito à vida e deveria ser garantido integralmente, independentemente de questões políticas, orçamentárias ou entraves burocráticos. Isso demonstra que os juízes vinham respeitando a indicação médica individual, a despeito do tratamento que estabelece a política pública. ${ }^{(14)}$

Tal postura, entretanto, vem sendo discutida em Fóruns Estaduais em Defesa da Saúde criados pelo Conselho Nacional de Justiça, sendo recomendada, inclusive, a adoção de medidas visando melhor subsidiar os magistrados e demais operadores do direito com informações clínicas, ou técnicas, para assegurar maior eficiência na solução das demandas judiciais envolvendo a assistência à saúde. ${ }^{(15)}$

A despeito dessa questão, é importante ressaltar, conforme exposto por Marques e Dallari(16), que à administração pública cabe elaborar uma política que possa atender, de forma integral e universal, ao direito à assistência farmacêutica, com base nas principais necessidades de saúde da população e nos recursos disponíveis, para que possa atender aos cidadãos de forma segura e equânime.

No que tange aos aspectos sanitários, deve-se considerar que, quando um medicamento é incorporado às políticas públicas, a responsabilidade do poder

\footnotetext{
${ }^{13}$ MARQUES, S.B. op. cit.

${ }^{14}$ Id. Ibid.

15 CONSELHO NACIONAL DE JUSTIÇA. Recomendação $n^{\circ}$ 31, de 30 de março de 2010. "Recomenda aos Tribunais a adoção de medidas visando a melhor subsidiar os magistrados e demais operadores do direito, para assegurar maior eficiência na solução das demandas judiciais envolvendo a assistência à saúde". Diário de Justiça Eletrônico n 61, 7 abr. 2010.

${ }^{16}$ MARQUES, S.B.; DALLARI, S.G. Garantia do direito social à assistência farmacêutica no Estado de São Paulo. Revista de Saúde Pública, São Paulo, v. 41, n.1, fev. 2007.
} 
público se amplifica, tendo em vista a massificação do seu uso. Nesse sentido, é preciso ofertar à população os medicamentos mais seguros (que sabidamente não provocam danos), eficazes (fazem o que se propõem a fazer), efetivos (fazem o que se propõem a fazer quando utilizados pelas pessoas em condições reais) e custos efetivos (entre as alternativas disponíveis, fazem o que se propõem a fazer para as pessoas em condições reais, ao menor custo). ${ }^{(17)}$

No caso das ações judiciais, surgem questionamentos sobre as evidências em que se baseiam a prescrição e a utilização das tecnologias em saúde. Trabalho que analisou características da demanda judicial de medicamentos antineoplásicos contra a Secretaria Estadual de Saúde de São Paulo, entre 2006 e 2007, concluiu pela concentração de prescrição em poucos profissionais e pela demanda para uso off label, para o qual, muitas vezes, não há evidências que suportem a utilização. ${ }^{(18)}$

Observa-se, pois, que há características específicas nas demandas judiciais por assistência farmacêutica que merecem ser analisadas sob o aspecto sanitário, principalmente no que se refere à segurança do paciente, em razão de possíveis prescrições inadequadas, mesmo que de medicamentos já selecionados e incorporados no SUS pela administração pública.

Especificamente com relação às demandas por medicamentos para uso off label, é possível que muitas dessas possam ser justificadas pela gravidade do caso, falta de resposta à terapia convencional e falta de opção terapêutica. Contudo, é importante lembrar que os profissionais prescritores estão, constantemente, sendo influenciados por propagandas de laboratórios que divulgam informações, por vezes distorcidas, sobre novas indicações para um medicamento já registrado, as quais são baseadas em estudos científicos que ficam aquém do padrão necessário para aprovação dos órgãos regulatórios. Nesses tipos de publicações, pretensamente científicas, profissionais desavisados avidamente incorporam o que lhes é "ensinado" por formadores de opinião, patrocinados direta ou indiretamente pela indústria farmacêutica. Isso, inclusive, representa uma manobra mercadológica das indústrias farmacêuticas para ampliarem o uso de seus produtos. ${ }^{(19),(20)}$

17 FALEIROS, R.F.; SATURNINO L.T.M.; ANDRADE, E.I.G.A.; SOUZA, A.L.C.; MARQUES, J.S.; GUERRA JUNIOR, A.A. Assistência Farmacêutica: o Reverso do Imperativo Tecnológico da Medicalização. In: AITH, Fernando et al. (Org.) Direito Sanitário: saúde e direito um diálogo possível. Belo Horizonte: Escola de Saúde Pública do Estado de Minas Gerais, 2010. p. 265-387.

${ }^{18}$ FIGUEIREDO, T.A.; PEPE, V.L.E.; OSORIO-DE-CASTRO, C.G.S. Um enfoque sanitário sobre a demanda judicial de medicamentos. Physis, Rio de Janeiro, v. 20, n. 1, 2010.

${ }^{19}$ PAULA, C. Silva. Uso off label de medicamentos: análise das demandas judiciais no CEMEPAR e conduta dos farmacêuticos no Paraná. 144 f. Dissertação (Mestrado em Ciências Farmacêuticas) Universidade Federal do Paraná, Curitiba, 2010.

${ }^{20}$ WANNMACHER, L. op.cit. 
Assim, conforme ressaltam Pepe et al.,(21) o uso da via judicial para a aquisição de medicamentos pode também favorecer a introdução e utilização de novas tecnologias de forma acrítica, cabendo destacar que parte dos medicamentos "novos" não representa real ganho em eficácia terapêutica, mas pode, inclusive, adicionar eventos adversos inesperados. Portanto, o uso de medicamentos sem registro sanitário ou fora das indicações para as quais foram registrados (uso off label) pode também significar riscos à saúde.

\section{Métodos}

Para investigar a ocorrência de uso de medicamento para indicações não aprovadas pela Anvisa, optou-se por utilizar um estudo transversal, exploratório com coleta retrospectiva de dados primários.

A fonte dos dados desta pesquisa foram os pareceres técnicos, denominados Notas Técnicas, referentes a solicitações de medicamento realizadas por ordem judicial, cuja ação foi movida pelo cidadão contra o Estado de Minas Gerais. Esses pareceres são emitidos por profissionais médicos e farmacêuticos da Assessoria Técnica da Secretaria de Estado de Saúde de Minas Gerais (AT/SES), setor responsável pelo cumprimento das ordens judiciais referentes a medicamentos, procedimentos médicos e outros produtos para a saúde, e se destinam a fornecer informações técnicas aos procuradores do Estado, na ocasião de manifestação no processo judicial.

Inicialmente foi feito o levantamento de todas as Notas Técnicas emitidas no período de janeiro a dezembro de 2010, sendo encontrado o total de 4.137. Foram excluídas aquelas referentes a solicitações extrajudiciais, ou seja, que não tratavam de demandas judiciais; as que tratavam de demandas judiciais por outros produtos e procedimentos para a saúde que não medicamentos; e as que não continham informações acerca do diagnóstico do paciente para o qual o medicamento foi demandado. Após as exclusões, foi identificado um total de 1.981 Notas Técnicas referentes a demandas judiciais por medicamentos, que é a amostra deste estudo. Os resultados foram avaliados por análise descritiva.

Esses documentos foram consultados para obter os seguintes dados: medicamento solicitado, patologia informada na ação judicial e concessão ou não de pedido de antecipação de tutela ou de liminar.

A verificação da indicação aprovada pela Anvisa foi realizada por meio de consulta à bula do medicamento registrado no Brasil, diretamente no site do fabricante na internet ou enviado por este, por e-mail, após solicitação. Foram

\footnotetext{
${ }^{21}$ PEPE, V.L.E.; FIGUEIREDO, T.A.; SIMAS, L.; OSÓRIO-DE-CASTRO, S.G.C.; VENTURA, M. A judicialização da saúde e os novos desafios da gestão da assistência farmacêutica. Ciência \& Saúde Coletiva, Rio de Janeiro, v. 15, n. 5, ago. 2010.
} 
consideradas off label todas as solicitações de medicamentos para uso em patologia não recomendada pela bula do laboratório fabricante, ou seja, cuja indicação de uso diverge da aprovada pela Anvisa.

A fim de possibilitar a comparação com outros estudos, os medicamentos foram classificados segundo o primeiro nível do Sistema Anatômico e Terapêutico Químico (código ATC), recomendado pala Organização Mundial da Saúde (OMS), que considera os sistemas ou órgãos nos quais as substâncias atuam.

Tendo em vista que o uso off label de medicamentos ocorre também quando há a prescrição do uso de um medicamento para faixa etária, dose ou, ainda, via de administração não recomendadas em bula, deve-se esclarecer que não foi objeto deste estudo investigar a ocorrência de solicitação de medicamento para uso nessas condições, mas somente a ocorrência de solicitação de medicamento para uso em patologia diferente da recomendada em bula, o que representa uma limitação desse estudo.

\section{IV.Resultados e discussão}

Inicialmente, apresentamos, na tabela a seguir, os medicamentos solicitados judicialmente à SES/MG, no período abordado no estudo, para uso em indicação não aprovada pela Anvisa, com o respectivo código ATC e o número de vezes em que foram pleiteados (Tabela 1).

No total de 1.981 Notas Técnicas analisadas, foram identificadas 212 solicitações de medicamentos para uso em patologia não recomendada pela bula do laboratório fabricante, realizadas por 196 pacientes.

Tendo em vista que não foram analisados os medicamentos para uso em faixa etária, dose e via de administração diferente da recomendada, o número de solicitações judiciais de medicamentos para uso off label pode ser ainda maior.

Pesquisa realizada por Silva Paula, (22) que analisou 934 solicitações de medicamentos conduzidas por ordem judicial à Secretaria de Estado de Saúde do Paraná, em 2008, encontrou que $12 \%$ estavam relacionadas com indicação diferente da recomendada ou off label quando comparadas com informações da Anvisa. Dessas, 43,75\% estavam relacionadas com o uso em patologia diferente daquela aprovada pelo órgão regulamentador.

A tabela, a seguir, apresenta os medicamentos mais solicitados para uso em indicação diferente da recomendada em bula aprovada pela Anvisa e as doenças para os quais eles foram demandados por ação judicial, em 2010 (Tabela 2).

\footnotetext{
${ }^{22}$ PAULA, C. Silva. op. cit.
} 
Tabela 1. Medicamentos solicitados pela via judicial à Secretaria de Estado de Saúde de Minas Gerais para uso em indicação off label e número de vezes em que foram pleiteados no ano de 2010.

\begin{tabular}{|c|c|c|}
\hline ATC* & Medicamento & $\mathrm{n}^{\star \star}$ \\
\hline $\mathrm{N}$ & ácido valproico & 9 \\
\hline G & alfafolitropina & 1 \\
\hline G & alfalutropina & 1 \\
\hline L & bevacizumabe & 23 \\
\hline G & cabergolina & 1 \\
\hline $\mathrm{J}$ & Cidofovir & 1 \\
\hline C & Clonidina & 3 \\
\hline G & danazol & 2 \\
\hline V & deferiprona & 2 \\
\hline $\mathrm{N}$ & divalproato de sódio & 1 \\
\hline $\mathrm{N}$ & gabapentina & 2 \\
\hline L & imatinibe & 1 \\
\hline $\mathrm{L}$ & linfliximabe & 8 \\
\hline $\mathrm{L}$ & irinotecano & 1 \\
\hline $\mathrm{N}$ & lamotrigina & 12 \\
\hline $\mathrm{L}$ & leflunomida & 1 \\
\hline$N$ & metilfenidato & 1 \\
\hline $\mathrm{L}$ & micofenolato de mofetila & 48 \\
\hline L & micofenolato de sódio & 1 \\
\hline A & miglustate & 1 \\
\hline $\mathrm{N}$ & olanzapina & 4 \\
\hline $\mathrm{N}$ & oxcarbazepina & 10 \\
\hline M & pamidronato dissódico & 5 \\
\hline D & pimecrolimo & 2 \\
\hline $\mathrm{N}$ & quetiapina & 16 \\
\hline $\mathrm{S}$ & ranibizumabe & 11 \\
\hline $\mathrm{L}$ & rituximabe & 17 \\
\hline G & sildenafila & 2 \\
\hline $\mathrm{L}$ & sirolimo & 7 \\
\hline A & sulfassalazina & 1 \\
\hline L & tacrolimo & 7 \\
\hline $\mathrm{D}$ & tacrolimo tópico & 1 \\
\hline $\mathrm{L}$ & tamoxifeno & 1 \\
\hline $\mathrm{N}$ & topiramato & 7 \\
\hline $\mathrm{N}$ & ziprasidona & 1 \\
\hline Total & & 212 \\
\hline
\end{tabular}

Fonte: Notas Técnicas referentes às solicitações de medicamentos realizadas por ordem judicial contra o Estado de Minas Gerais, em 2010.

*De acordo com a OMS, o primeiro nível do código ATC corresponde aos sistemas ou órgãos onde as substâncias atuam: A - Sistema digestivo e metabolismo; B - Sangue e órgãos hematopoéticos; C - Sistema cardiovascular; D - Medicamentos e produtos dermatológicos; G - Sistema genito-urinário e hormônios sexuais; H - Hormônios sistêmicos, excluindo-se hormônios sexuais; J - Anti-infecciosos gerais para uso sistêmico; L - Agentes antineoplásicos e imunomoduladores; $\mathrm{M}$ - Sistema músculo-esquelético; $\mathrm{N}$ - Sistema nervoso; $\mathrm{P}$ - Antiparasitários, inseticidas e repelentes; $\mathrm{R}$ - Sistema respiratório; S - Órgãos sensoriais; e V - Vários.

${ }^{\star *} \mathrm{O}$ n refere-se ao número de vezes em que o medicamento foi solicitado para uso off label. 
Tabela 2. Os três medicamentos mais solicitados pela via judicial à Secretaria de Estado de Saúde de Minas Gerais para uso em indicação diferente da recomendada de acordo com a Anvisa e patologias informadas nas ações judiciais no ano de 2010.

\begin{tabular}{|c|c|c|}
\hline Medicamento & Doença informada na ação judicial & $\mathrm{n}^{*}$ \\
\hline bevacizumabe & Câncer cerebral & 3 \\
\hline bevacizumabe & Coriorretinopatia fibromacular idiopática & 1 \\
\hline bevacizumabe & Degeneração macular relacionada a idade & 5 \\
\hline bevacizumabe & Deslocamento de retina & 2 \\
\hline bevacizumabe & Distrofia hereditária da retina & 1 \\
\hline bevacizumabe & Edema macular & 2 \\
\hline bevacizumabe & Glaucoma neovascular & 1 \\
\hline bevacizumabe & Maculopatia distrófica e exsudativa & 1 \\
\hline bevacizumabe & Retinopatia diabética & 6 \\
\hline bevacizumabe & Trombose ocular & 1 \\
\hline micofenolato de mofetila & Esclerose sistêmica & 1 \\
\hline micofenolato de mofetila & Glomerulonefrite membrano proliferativa & 1 \\
\hline micofenolato de mofetila & Granulomatose de Wegener & 2 \\
\hline micofenolato de mofetila & Hepatite autoimune & 3 \\
\hline micofenolato de mofetila & Insuficiência renal crônica & 1 \\
\hline micofenolato de mofetila & Lúpus eritematoso sistêmico & 26 \\
\hline micofenolato de mofetila & Miastenia gravis & 1 \\
\hline micofenolato de mofetila & Pênfigo foliáceo & 1 \\
\hline micofenolato de mofetila & Pênfigo vulgar & 1 \\
\hline micofenolato de mofetila & Síndrome miastênica & 1 \\
\hline micofenolato de mofetila & Síndrome nefrótica & 3 \\
\hline micofenolato de mofetila & Transplante de medula óssea & 1 \\
\hline micofenolato de mofetila & Transplante de medula óssea & 2 \\
\hline micofenolato de mofetila & Transplante de pâncreas & 2 \\
\hline micofenolato de mofetila & Transplante de pulmão & 1 \\
\hline micofenolato de mofetila & Vasculite Leucocitoclástica Idiopática & 1 \\
\hline rituximabe & Esclerose múltipla & 1 \\
\hline rituximabe & Esclerose sistêmica & 2 \\
\hline rituximabe & Granulomatose de Wegener & 1 \\
\hline rituximabe & Lúpus eritematoso sistêmico & 8 \\
\hline rituximabe & Macroglobulinemia de Waldenström & 1 \\
\hline rituximabe & Miastenia gravis & 1 \\
\hline rituximabe & Neuromielite ótica & 1 \\
\hline rituximabe & Polimiosite & 1 \\
\hline rituximabe & Trombocitemia imunológica primária & 1 \\
\hline
\end{tabular}

Fonte: Notas Técnicas referentes às solicitações de medicamentos realizadas por ordem judicial contra o Estado de Minas Gerais, em 2010.

*O n representa o número de vezes em que o medicamento foi solicitado para a mesma indicação. 
Conforme pode ser observado, os três medicamentos mais solicitados para uso em indicação diferente da recomendada em bula aprovada pela Anvisa foram o micofenolato de mofetila, o bevacizumabe e o rituximabe, que representaram, respectivamente, $23 \%, 11 \%$ e $8 \%$ das solicitações de medicamentos nessa condição.

Diante dessa constatação, cabe citar que em estudo realizado por Lopes et al., ${ }^{(23)}$ que avaliou a racionalidade das ações judiciais e pedidos administrativos por medicamentos antineoplásicos com maior impacto financeiro para o SUS, recebidos pela Secretaria de Estado da Saúde de São Paulo, bevacizumabe $(59,5 \%)$ e rituximabe $(31 \%)$ foram os medicamentos com maior percentual de solicitações sem evidência científica de eficácia e segurança, nos anos estudados.

Os três medicamentos mais solicitados no presente estudo para uso em indicação off label, bevacizumabe, rituximabe e micofenolato de mofetila, são comercializados no Brasil pelo Laboratório Roche. Dentre esses, apenas o fármaco micofenolato de mofetila é comercializado também por outros laboratórios farmacêuticos na forma de medicamento genérico.

As indicações previstas na bula dos medicamentos contendo o princípio ativo micofenolato de mofetila correspondem à profilaxia e tratamento de pacientes submetidos a transplante de rim, coração ou fígado. O fármaco bevacizumabe é indicado no tratamento de pacientes portadores de carcinoma metastático do cólon ou reto, câncer de pulmão metastático, câncer de mama metastático e câncer de células renais metastático e/ou avançado. Já o medicamento rituximabe, que foi aprovado e registrado no órgão de regulação sanitária nacional, inicialmente, para o tratamento antineoplásico (tratamento do câncer) e, em seguida, para tratamento da artrite reumatoide, atualmente está indicado também para o tratamento da leucemia linfoide crônica. ${ }^{(24)}$

Não há, portanto, indicação formal, aprovada e regulamentada pela Anvisa para uso dos medicamentos micofenolato de mofetila, bevacizumabe e rituximabe no tratamento das doenças informadas na ação judicial que estão relacionadas na Tabela 2, assim como o uso desses medicamentos no tratamento dessas doenças não é recomendado pelo laboratório fabricante, como pode ser observado no documento legal desses produtos (a bula).

Observa-se, naquela tabela, expressiva solicitação dos medicamentos micofenolato de mofetila e rituximabe para uso off label no tratamento do lúpus

\footnotetext{
${ }^{23}$ LOPES, L.C.; BARBERATO-FILHO, S.; COSTA, A.C.; OSORIO-DE-CASTRO, C.G.S. Uso racional de medicamentos antineoplásicos e ações judiciais no estado de São Paulo. Revista de Saúde Pública, São Paulo, v. 44, n. 4, ago. 2010.

${ }^{24}$ Informações disponibilizadas pelo laboratório fabricante nas respectivas bulas dos medicamentos. ROCHE DO BRASIL. Bula dos produtos. Disponível em: <http://www.roche.com.br/portal/ rochebrazil/lista_de_produtos?paf_dm=full\&categoryld=re7193008_ct57043>. Acesso em: 10 mai 2011.
} 
eritematoso sistêmico, uma doença inflamatória crônica, multissistêmica, de causa desconhecida e que possui natureza autoimune.

A despeito de controvérsias quanto ao uso dos medicamentos micofenolato de mofetila e rituximabe no tratamento do lúpus, os resultados dessa investigação apontam para possível uso rotineiro desses medicamentos no tratamento dessa doença na prática clínica. Diante disso, vale enfatizar que, uma vez que tais usos não se encontram completamente estabelecidos, um processo documentado visando consentimento para o tratamento, junto ao paciente, é recomendável. Isso inclui obter informações adicionais sobre as incertezas e discutir com o paciente, ou um responsável por este, a razão para a utilização do medicamento, terapias alternativas e possíveis reações adversas. ${ }^{(25)}$ Vale enfatizar, ainda, que o tratamento do lúpus eritematoso sistêmico está previsto no SUS, por meio do Componente Especializado da Assistência Farmacêutica (CEAF), com o fornecimento de vários medicamentos, os quais devem ser avaliados pelo médico assistente do paciente.

O CEAF é uma estratégia de acesso a medicamentos no âmbito do SUS, caracterizado pela busca da garantia da integralidade do tratamento medicamentoso, em nível ambulatorial, cujas linhas de cuidado estão definidas nos Protocolos Clínicos e Diretrizes Terapêuticas publicados pelo Ministério da Saúde. Na lista dos medicamentos desse componente estão incluídos, sobretudo, aqueles destinados ao tratamento de condições raras ou de baixa prevalência ou, ainda, que possuam custo unitário elevado ou alto custo de tratamento. $\mathrm{O}$ acesso aos medicamentos que fazem parte das linhas de cuidado para as doenças contempladas no âmbito do CEAF é garantido mediante a pactuação entre a União, Estados e Municípios, conforme as diferentes responsabilidades definidas na Portaria MS $n^{\circ} 2981 / 09$, que regulamenta o programa. ${ }^{(26)}$

No caso do medicamento micofenolato de mofetila, por exemplo, o seu fornecimento por meio do CEAF se encontra autorizado apenas aos pacientes transplantados, que apresentem as seguintes condições clínicas: rim transplantado, coração transplantado, fígado transplantado ou, ainda, falência ou rejeição de transplante de rim. Essas indicações englobam todas as previstas na bula do medicamento pleiteado e, portanto, autorizadas pela Anvisa.

É oportuno salientar que o registro de um produto farmacêutico pela Anvisa significa apenas que aquele medicamento atende aos requisitos mínimos de segurança biológica e eficácia terapêutica estabelecidos pela agência reguladora para a finalidade estudada, representando que seu fabricante, a partir

\footnotetext{
${ }^{25}$ GAZARIAN, M.; KELLY, M.; MCPHEE, J.R.; GRAUDINS, L.V.; WARD, R.L; CAMPBELL, T.J. op. cit. ${ }^{26}$ BRASIL. Portaria n ${ }^{\circ}$ 2.981, de 26 de novembro de 2009. "Aprova o Componente Especializado da Assistência Farmacêutica". Diário Oficial [da] Republica Federativa do Brasil, Brasília, DF, 30 nov. 2009. Disponível em: <http://portal2.saude.gov.br/saudelegis/LEG_NORMA_PESQ_CONSULTA. CFM>. Acesso em: 12 ago. 2011.
} 
de então, tem o direito de comercializá-lo. Isso não implica, obrigatoriamente, a sua incorporação e fornecimento no âmbito do sistema público de saúde, inclusive, porque o processo de registro não considera a comparação entre as alternativas terapêuticas disponíveis. ${ }^{(27)}$ Assim, o fato de o medicamento possuir registro junto à Anvisa não o aprova automaticamente para fornecimento por meio das políticas públicas de fornecimento de medicamentos, sendo necessária sua devida regulamentação para esse fim. Essas políticas consideram como critérios de incorporação de medicamentos, além de fatores como a indicação reconhecida e autorizada pelo órgão sanitário responsável, a existência de estudos que comprovem a superioridade do medicamento para a finalidade desejada em comparação com as alternativas terapêuticas existentes, as relações risco/ benefício e custo/benefício desses medicamentos, entre outros.

Além do CEAF, compõem os programas de assistência farmacêutica do SUS os Componentes Estratégico e Básico. O Componente Estratégico da Assistência Farmacêutica tem financiamento exclusivo do Ministério da Saúde, sendo destinado às ações de assistência farmacêutica de programas específicos, considerados estratégicos para a saúde, como o controle de endemias, tais como a tuberculose, a hanseníase, a malária, a leishmaniose, a doença de chagas e outras doenças endêmicas de abrangência nacional ou regional; antirretrovirais do programa DST/AIDS; sangue e hemoderivados; imunobiológicos; medicamentos e insumos para combate ao tabagismo e para alimentação e nutrição.

O Componente Básico da Assistência Farmacêutica, regulamentado pela Portaria MS/GM n 4.217/10, ${ }^{(28)}$ por sua vez, destina-se à aquisição de medicamentos e insumos da assistência farmacêutica no âmbito da atenção básica à saúde e àqueles relacionados a agravos e programas de saúde específicos desse nível de atenção.

O Gráfico 1 apresenta os percentuais de medicamentos solicitados para uso off label de acordo com a inclusão, ou não, nos programas de assistência farmacêutica do SUS.

Considerando todos os medicamentos pleiteados para uso em indicação diferente da recomendada, no período analisado, 46\% não estavam incluídos em programas de assistência farmacêutica do SUS, $51 \%$ estavam incluídos no Componente Especializado e 3\% estavam incluídos no Componente Básico (Gráfico 1).

\footnotetext{
${ }^{27}$ MINISTÉRIO DA SAÚDE. Secretaria de Atenção à Saúde/Departamento de Atenção Especializada/ Coordenação Geral de Média e Alta Complexidade. Nota Técnica 2010: o acesso a medicamentos antineoplásicos no SUS. 2010. Disponível em: <http://portal.saude.gov.br/portal/arquivos/pdf/ cgmac_medicamentos.pdf>. Acesso em: $10 \mathrm{dez} .2011$.

${ }^{28}$ MINISTÉRIO DA SAÚDE. Portaria $n^{\circ} 4.217$, de 28 de dezembro de 2010. "Aprova as normas de financiamento e execução do Componente Básico da Assistência Farmacêutica.”. Disponível em: $<\mathrm{http}: / /$ portal.saude.gov.br/portal/arquivos/pdf/Portaria_MS_4217_28_12_2010.pdf>. Acesso em: 29 mar. 2013.
} 


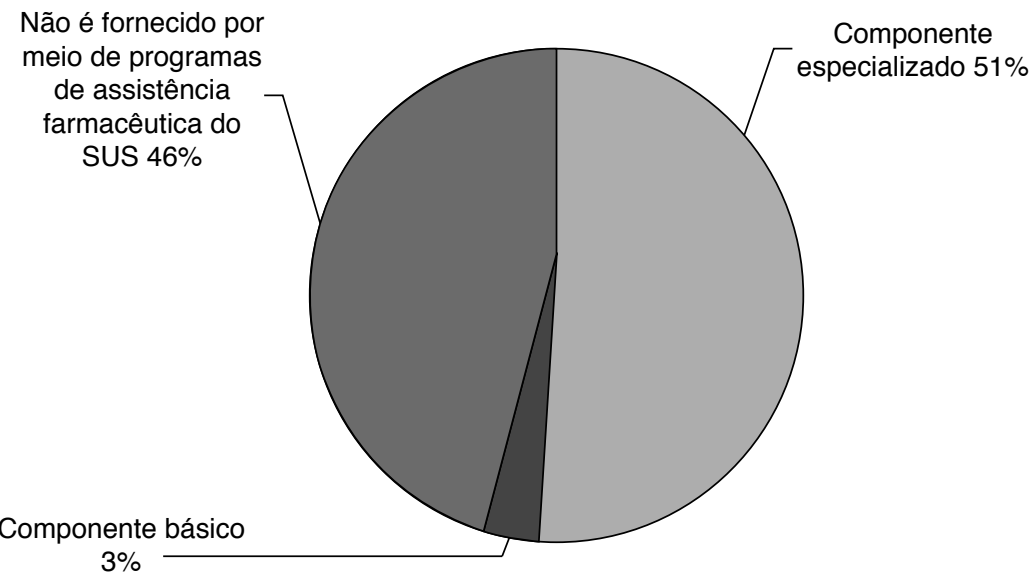

Fonte: Notas Técnicas referentes às solicitações de medicamentos realizadas por ordem judicial contra o Estado de Minas Gerais, em 2010.

Gráfico 1. Presença, em programas de assistência farmacêutica do SUS, dos medicamentos solicitados por meio de ações judiciais à Secretaria de Estado de Saúde de Minas Gerais no ano de 2010 para uso em patologia diferente da recomendada em bula.

Em consonância com o apontado em estudo sobre a judicialização do acesso a medicamentos no Estado de Minas Gerais, realizado por Machado et al., ${ }^{(29)}$ observou-se expressiva solicitação judicial de medicamentos de alto custo, que, atualmente, estão incluídos no programa de medicamentos do CEAF. Segundo esses autores, tais medicamentos são caros, o que pode ser a principal motivação dos pacientes para o ingresso de ações judiciais. Além disso, o acesso a eles, pelo programa, exige o cumprimento dos critérios de inclusão estabelecidos nos Protocolos Clínicos e Diretrizes Terapêuticas do Ministério da Saúde.

Ressalta-se que, em se tratando de solicitação de medicamentos para uso off label, o acesso é negado, tendo em vista que um dos critérios para a padronização dos medicamentos oferecidos por meio do CEAF é a comprovação de eficácia e segurança de uso do medicamento para determinada condição clínica, assegurada por estudos clínicos robustos e confiáveis. No caso de uso em indicação off label, muitas vezes, tal comprovação não existe, o que o exclui dos Protocolos Clínicos e Diretrizes Terapêuticas aprovados pelo Ministério da Saúde.

Os Protocolos Clínicos e Diretrizes Terapêuticas são instrumentos legais, legitimados por portarias específicas, de orientação para o uso racional de

\footnotetext{
${ }^{29}$ MACHADO, M.A.A.; ACURCIO, F.A.; BRANDÃO, C.M.R.; FALEIROS, D.R.; GUERRA JUNIOR, A.A.; CHERCHIGLIA, M.L.; ANDRADE, E.I.G. Judicialização do acesso a medicamentos no Estado de Minas Gerais, Brasil. Revista de Saúde Pública, São Paulo, v. 45, n. 3, jun. 2011.
} 
medicamentos. Segundo Marin et al., ${ }^{(30)}$ o uso racional de medicamento é um conjunto de práticas que inclui, entre outros, a escolha terapêutica adequada, dose, administração e duração do tratamento apropriados e a inexistência de contraindicação e mínima probabilidade de reações adversas.

É oportuno mencionar que, recentemente, a fim de estabelecer os limites da assistência farmacêutica a ser prestada no âmbito do SUS, bem como as regras de incorporação de tecnologias no sistema, o Congresso Brasileiro aprovou a Lei $n^{\circ} 12.401 / 11^{(31)}$, que alterou a Lei $n^{\circ} 8.080 / 90$. Entre as mudanças aprovadas, está a de que a integralidade da assistência terapêutica a ser prestada no âmbito do SUS tem por limites a dispensação de medicamento prescrito em conformidade com as diretrizes terapêuticas estabelecidas para o agravo à saúde a ser tratado, instituídas pelo gestor federal do SUS, em protocolo clínico específico. $\mathrm{Na}$ ausência de protocolo clínico, a dispensação limitar-se-á aos produtos constantes nas relações de medicamentos elaboradas pelos gestores do SUS. Define, ainda, que a incorporação, a exclusão ou alteração de medicamentos, produtos e procedimentos disponibilizados no SUS, bem como a constituição ou a alteração de protocolo clínico ou de diretriz terapêutica, são atribuições do Ministério da Saúde, assessorado pela Comissão Nacional de Incorporação de Tecnologias no SUS.

Segundo Marques, ${ }^{(32)}$ a redução do princípio constitucional de integralidade de assistência à saúde à oferta pública dos medicamentos selecionados pelo gestor público representa um retrocesso em relação ao cumprimento amplo dos direitos humanos, focado em princípios que permitem a garantia digna da saúde de todos, uma vez que os protocolos e listas oficiais não contemplam todas as necessidades de saúde da população, bem como podem apresentar falhas e demora em sua atualização, de acordo com novas tecnologias e recursos disponíveis.

Diante desse embate, é importante salientar que a Lei $\mathrm{n}^{\circ} 12.401$ acertou em condicionar a oferta de medicamentos à devida análise prévia de comprovação de efetividade, pois deve-se atentar para o fato de que a incorporação de um medicamento em um programa de assistência farmacêutica do SUS significa propiciar o acesso de milhares de pessoas a esse medicamento. Sendo assim, pode-se dizer que um medicamento distribuído sem a observância de todos os requisitos que garantem a sua qualidade, segurança e eficácia pode gerar danos

\footnotetext{
${ }^{30}$ MARIN, N.; LUIZA, V.L.; SERPA, C.G; CASTRO, O; SANTOS, O. M. Assistência Farmacêutica para gerentes municipais 2003. Disponível em: <http://www.opas.org.br/medicamentos/site/ UploadArq/0080.pdf>. Acesso em: 05 out. 2011.

${ }^{31}$ BRASIL. Lei $n^{\circ} 12.401$, de 28 de abril de 2011. "Altera a Lei no 8.080, de 19 de setembro de 1990, para dispor sobre a assistência terapêutica e a incorporação de tecnologia em saúde no âmbito do Sistema Único de Saúde - SUS”. Diário Oficial [da] Republica Federativa do Brasil, Brasília, DF, 29 abr. 2011. Disponível em <http://www.planalto.gov.br/ccivil_03/_Ato2011-2014/2011/Lei/L12401. htm>. Acesso em: 12 ago. 2011.

${ }^{32}$ MARQUES, S.B. op. cit.
} 
decorrentes de riscos até então não previstos nas populações dos estudos, que, geralmente, até por critérios de segurança, são reduzidas a um número menor de indivíduos.

Contudo, o perigo pode estar na demora de incorporação de tecnologias pelo SUS, que pode privar o cidadão de ter acesso aos melhores tratamentos ofertados pelo progresso da ciência. Cabe ressaltar, ainda, a existência de muitos brasileiros cujas características de suas doenças não os enquadram em protocolos clínicos do SUS, aos quais deve ser dado um tratamento diferenciado.

Assim, não se trata de contrapor à existência dos protocolos clínicos e à necessidade de avaliação da relação custo-benefício dos tratamentos padronizados para disponibilização no SUS, mas de enfatizar a necessidade de análise daqueles casos especiais de pacientes que não podem ser atendidos, exclusivamente, com base na lista de medicamentos constantes nesses instrumentos.

A seguir, na Tabela 3, é apresentada a classificação dos medicamentos solicitados judicialmente à Secretaria de Estado de Saúde de Minas Gerais para uso em indicação off label, de acordo com a classe terapêutica e frequência das solicitações.

Tabela 3. Classificação dos medicamentos solicitados judicialmente à Secretaria de Estado de Saúde de Minas Gerais para uso em indicação off label, segundo a classe terapêutica e frequência das solicitações, no ano de 2010.

\begin{tabular}{lcc}
\hline Classe terapêutica (ATC) & Frequência (\%) & $\mathrm{n}^{\star}$ \\
\hline L - Agentes antineoplásicos e imunomoduladores & 54,25 & 115 \\
N - Sistema nervoso & 29,72 & 63 \\
S - Órgãos sensoriais & 5,19 & 11 \\
G - Sistema gênito-urinário e hormônios sexuais & 3,30 & 7 \\
M - Sistema músculo esquelético & 2,36 & 5 \\
C - Sistema cardiovascular & 1,42 & 3 \\
D - Medicamentos e Produtos Dermatológicos & 1,42 & 3 \\
A - Sistema digestivo e metabolismo & 0,94 & 2 \\
V- Vários & 0,94 & 2 \\
J - Anti-infecciosos gerais para uso sistêmico & 0,47 & 1 \\
\hline
\end{tabular}

Fonte: Notas Técnicas referentes às solicitações de medicamentos realizadas por ordem judicial contra o Estado de Minas Gerais, em 2010.

*O n representa o número de vezes que o medicamento da respectiva classe terapêutica foi solicitado para uso off label.

Considerando todos os medicamentos solicitados para uso em indicação off label relacionados no presente estudo, a classe terapêutica, de acordo com o código 
ATC, mais solicitada foi a dos agentes antineoplásicos e imunomoduladores, que correspondeu a $54,20 \%$ das solicitações (Tabela 3). Essa mesma classe terapêutica foi a mais solicitada para uso off label também no estudo realizado por Silva Paula. ${ }^{(33)}$ A autora salientou que o fornecimento desses medicamentos por meio de demandas judiciais para casos não previstos nos protocolos clínicos retira recursos destinados, principalmente, à atenção de média complexidade e direciona para terapias que apresentam programas próprios, ou para terapias ainda sem comprovação científica.

Destaca-se que os medicamentos antineoplásicos não são fornecidos pelos programas ambulatoriais de assistência farmacêutica do SUS (Componentes Básicos, Estratégico e Especializado). Isso porque, com base na Política Nacional de Atenção Oncológica, instituída pela Portaria GM/MS n².439/05, ${ }^{\left({ }^{(3)}\right.}$ o tratamento do câncer é realizado por meio de um programa específico no SUS, que estabeleceu os Centros/Unidades de Alta Complexidade em Oncologia (Cacons/Unacons). Esses estabelecimentos visam ao atendimento específico dos pacientes portadores de neoplasia maligna, cabendo-Ihes o fornecimento de medicamentos indicados e prescritos por seus profissionais. Vale mencionar que os Cacons/Unacons são ressarcidos pelo Governo Federal, na forma da legislação vigente, do desembolso dos recursos necessários ao atendimento, incluindo o fornecimento dos medicamentos necessários aos pacientes portadores de câncer. ${ }^{(35)}$

Segundo Lopes et al., ${ }^{(36)}$ a área oncológica apresenta grande demanda pela via judicial, muito provavelmente, devido ao custo elevado e à sofisticação tecnológica dos tratamentos. Esses mesmos autores avaliaram a racionalidade das ações judiciais e pedidos administrativos recebidos pela Secretaria de Estado da Saúde de São Paulo de medicamentos antineoplásicos com maior impacto financeiro para o SUS em 2006 e 2007, segundo evidências científicas de eficácia e segurança. Aproximadamente $17 \%$ dos pedidos não tinham evidência para a indicação mencionada no pleito. A partir dos resultados encontrados, os autores ressaltaram a necessidade de qualificação técnica para tratar as demandas judiciais e de capacitação dos profissionais no manejo da literatura científica, na seleção adequada dos fármacos e na escolha da melhor conduta terapêutica para cada condição clínica, para o acesso a tecnologias eficazes e

\footnotetext{
${ }^{33}$ PAULA, C. Silva. op. cit.

${ }^{34}$ MINISTÉRIO DA SAÚDE. Portaria GM/MS n².439, de 8 de dezembro de 2005. "Institui a Política Nacional de Atenção Oncológica: Promoção, Prevenção, Diagnóstico, Tratamento, Reabilitação e Cuidados Paliativos, a ser implantada em todas as unidades federadas, respeitadas as competências das três esferas de gestão". Disponível em: <http://www.saude.mg.gov.br/atos_ normativos/legislacao-sanitaria/estabelecimentos-de-saude/oncologia/Portaria2439.pdf>. Acesso em: 29 mar. 2013.

${ }^{35}$ MINISTÉRIO DA SAÚDE. Secretaria de Atenção à Saúde/Departamento de Atenção Especializada/ Coordenação Geral de Média e Alta Complexidade. Nota Técnica 2010: o acesso a medicamentos antineoplásicos no SUS. 2010, cit.

${ }^{36}$ LOPES, L. C.; BARBERATO-FILHO, S.; COSTA, A. C.; OSORIO-DE-CASTRO, C.G.S. op. cit.
} 
seguras e aprimoramento do modelo de assistência farmacêutica em oncologia.

Diante dessa observação, para ressaltar a necessidade de instrução técnica para tratar as demandas judiciais por medicamentos, cabe mencionar que, no presente estudo, o fornecimento imediato de $78,30 \%$ dos medicamentos solicitados para uso off label foi determinado por meio de liminar judicial ou de antecipação de tutela (Tabela 4).

Tabela 4. Decisões judiciais sobre a concessão dos medicamentos para uso off label solicitados à Secretaria de Estado de Saúde de Minas Gerais no ano de 2010

\begin{tabular}{lcc}
\hline Decisão judicial sobre a concessão do medicamento & $\mathrm{n}^{*}$ & Frequência (\%) \\
\hline Liminar ou antecipação de tutela deferida & 166 & 78,3 \\
Liminar ou antecipação de tutela indeferida & 23 & 10,85 \\
Não foi encontrada informação sobre a concessão ou não & 23 & 10,85 \\
da liminar ou da antecipação de tutela na Nota Técnica. & & \\
\hline
\end{tabular}

Fonte: Notas Técnicas referentes às solicitações de medicamentos realizadas por ordem judicial contra o Estado de Minas Gerais, em 2010.

${ }^{*} \mathrm{O} n$ refere-se ao número de medicamentos solicitados para uso off label.

A partir desses resultados, observa-se uma tendência, quase absoluta, também observada em outros estudos, ${ }^{(37),(38)}$ do Poder Judiciário em conceder os medicamentos que são pleiteados.

Sobre a concessão de medicamentos por meio de liminares ou de antecipação de tutela, Vieira(39) chama atenção para os casos nos quais, posteriormente, se chega à conclusão de que a demanda não se justificava, resultando no desperdício de recursos públicos.

No entanto, não se pode deixar de considerar a importância da antecipação de tutela para evitar danos graves e irreversíveis à vida do paciente, caso o medicamento solicitado não seja fornecido tempestivamente, conforme apontado pelo Ministro do Supremo Tribunal Federal Gilmar Mendes, na ocasião de análise da Suspensão de Tutela Antecipada $n^{\circ} 145 .{ }^{(40)}$

Segundo apontado por Pepe et al.,(41) entre algumas características comuns nas ações judiciais relacionadas ao direito à saúde, identificadas nos estudos realizados em diferentes regiões do país, está o fato de a maioria ser deferida

\footnotetext{
${ }^{37}$ PEPE, V.L.E.; FIGUEIREDO, T.A.; SIMAS, L.; OSÓRIO-DE-CASTRO, S.G.C.; VENTURA, M. op. cit.

${ }^{38}$ VIEIRA, F.S. Ações judiciais e direito à saúde: reflexão sobre a observância aos princípios do SUS. Revista de Saúde Pública, São Paulo, v. 42, n. 2, abr. 2008.

${ }^{39}$ Id. Ibid.

40 SUPREMO TRIBUNAL DE JUSTIÇA. STA 175 AgR/ CE - CEARÁ AG. REG. NA SUSPENSÃO DE TUTELA ANTECIPADA. Relator: Min. Gilmar Mendes. Julgamento em: 17/03/2010. Diário de Justiça Eletrônico. N. 76, 30 abr. 2010. Disponível em: <http://redir.stf.jus.br/paginadorpub/ paginador.jsp?docTP=AC\&docID=610255>. Acesso em: 24 out. 2012.

${ }^{41}$ PEPE, V.L.E.; FIGUEIREDO, T.A.;SIMAS, L.; OSÓRIO-DE-CASTRO, S.G.C.; VENTURA, M. op. cit.
} 
tendo como praticamente única base a prescrição medicamentosa apresentada pelo reivindicante. Diante disso, vale alertar que, no caso de medicamentos para uso off label, há um risco sanitário a ser considerado. Esse é um dos motivos que apontam para a importância de os juízes estarem bem orientados tecnicamente na ocasião de análise dos pedidos judiciais por medicamentos.

A respeito desse tema, a Recomendação $n^{\circ} 31 / 10^{(42)}$ do Conselho Nacional Justiça orientou os tribunais a adotar medidas visando melhor subsidiar os magistrados e demais operadores de direito, para assegurar maior eficiência na solução das demandas judiciais envolvendo a assistência à saúde. Entre essas medidas, está a de celebrar convênios que objetivem disponibilizar apoio técnico, composto por médicos e farmacêuticos, para auxiliar os magistrados na formação de um juízo de valor quanto à apreciação das questões clínicas apresentadas pelas partes das ações relativas à saúde.

Segundo essa recomendação, em audiência pública realizada pelo Supremo Tribunal Federal para discutir as questões relativas às demandas judiciais que objetivam o fornecimento de prestações de saúde, foi constatada a carência de informações clínicas prestadas aos magistrados a respeito dos problemas de saúde enfrentados pelos autores dessas demandas. Isso demonstra que o próprio judiciário ressalta a importância de se considerarem medidas técnicas na análise de casos concretos.

Pepe et al., ${ }^{(43)}$ em estudo que abordou os principais elementos de interferência da "judicialização de medicamentos" no ciclo da Assistência Farmacêutica, apontaram mecanismos possíveis de serem adotados na tomada de decisão nesse âmbito pelos gestores e profissionais do sistema de justiça, para garantir a segurança e a proteção dos usuários e contribuir para a efetivação do direito à saúde.

A proposta para análise das demandas judiciais na tomada de decisão sobre o fornecimento de medicamentos leva em consideração que devem ser fornecidos os medicamentos que possuam as melhores evidências disponíveis sobre eficácia e segurança. No caso de medicamento para uso off label, deve-se evitar esse tipo de prescrição, que pode trazer riscos aos pacientes, especialmente quando não foram realizados ensaios clínicos a fim de confirmar a eficácia e a segurança do medicamento para determinado uso. Também, nesse caso, deve-se questionar a segurança sanitária do medicamento e seguir na análise buscando-se uma alternativa terapêutica disponível e segura. No entanto, não havendo alternativa terapêutica no SUS para a condição patológica apresentada pelo paciente, ou caso esse já a tenha utilizado e não tenha apresentado resposta terapêutica, e, ainda, havendo evidências

\footnotetext{
${ }^{42}$ CONSELHO NACIONAL DE JUSTIÇA. Recomendação $n^{\circ} 31$, de 30 de março de 2010. op.cit. ${ }^{43}$ PEPE, V.L.E.; FIGUEIREDO, T.A.; SIMAS, L.; OSÓRIO-DE-CASTRO, S.G.C.; VENTURA, M. op. cit.
} 
científicas para a conduta, deve-se considerar a possibilidade da necessidade de fornecimento do medicamento. ${ }^{(44)}$

\section{Considerações Finais}

Acompanhando a tendência encontrada em vários outros estudos, o uso off label de medicamentos por meio de ações judiciais é uma realidade observada na Secretaria de Estado de Saúde de Minas Gerais. Esse fato aponta para a necessidade de maior cautela do Poder Judiciário na ocasião de análise das solicitações de medicamentos, tendo em vista não só o comprometimento do acesso universal às ações e serviços públicos de saúde em favor do atendimento individual, mas também os riscos sanitários envolvidos.

Trata-se de uma questão complexa, que exige esforço, tanto por parte do Poder Judiciário quanto por parte dos gestores do SUS, para trabalharem juntos na solução das demandas. Assim, o judiciário deve continuar na busca pelo entendimento das questões técnicas que norteiam a "judicialização da saúde" e os gestores devem evoluir na busca pela melhoria da assistência farmacêutica prestada, com base nos critérios de uso racional de medicamentos, sem, entretanto, deixar de atender aos casos de fornecimento de medicamentos que fogem aos Protocolos Clínicos, mas que, pelo avanço da ciência e por suas peculiaridades, se mostrarem necessários.

\section{Referências}

AITH, F. Curso de direito sanitário: a proteção do direito à saúde no Brasil. São Paulo: Quartier Latin, 2007.

; DALLARI, S.G. Vigilância em saúde no Brasil: os desafios dos riscos sanitários do século XXI e a necessidade de criação de um sistema nacional de vigilância em saúde. Revista de Direito Sanitário, São Paulo, v. 10, n. 2, jul./ out. 2009.

FALEIROS, R.F.; SATURNINO, L.T.M.; ANDRADE, E.I.G.A.; SOUZA, A.L.C.; MARQUES, J.S.; GUERRA JUNIOR, A.A. Assistência farmacêutica: o reverso do imperativo tecnológico da medicalização. In: AITH, Fernando et al. (Org.) Direito sanitário: saúde e direito um diálogo possível. Belo Horizonte: Escola de Saúde Pública do Estado de Minas Gerais, 2010. p. 265-387.

${ }^{44}$ PEPE, V.L.E.; FIGUEIREDO, T.A.; SIMAS, L.; OSÓRIO-DE-CASTRO, S.G.C.; VENTURA, M. op. cit. 
FIGUEIREDO, T.A.; PEPE, V.L.E.; OSORIO-DE-CASTRO, C.G.S. Um enfoque sanitário sobre a demanda judicial de medicamentos. Physis, Rio de Janeiro, v. 20, n. 1, 2010.

GAZARIAN, M. Off label use of medicines in the paediatric population: recommendations for assessing appropriateness. Sydney 7 May 2007. 25p. Disponível em: <http://archives.who.int/eml/expcom/children/ttems/WHOofflabel pdf>. Acesso em: 22 out. 2012.

; KELLY, M.; MCPHEE, J.R.; GRAUDINS, L.V.; WARD, R.L.; CAMPBELL, T.J. Off-label use of medicines: consensus recommendations for evaluating appropriateness. The Medical Journal of Australia, Austrália, v.185, n.10, p. 544-547, Nov. 2006.

KAIRUZ, T.E.; GARGIULO, D.; BUNT, C.; GARG, S. Quality, Safety and Efficacy in the 'Off-Label' Use of Medicines. Current Drug Safety, United Arab Emirates, v. 2, n. 1, p. 89-95, Jan. 2007.

LOPES, L.C.; BARBERATO-FILHO, S.; COSTA, A.C.; OSORIO-DE-CASTRO, C.G.S. Uso racional de medicamentos antineoplásicos e ações judiciais no Estado de São Paulo. Revista de Saúde Pública, São Paulo, v. 44, n. 4, ago. 2010.

MACHADO, M.A.A.; ACURCIO, F.A.; BRANDÃO, C.M.R.; FALEIROS, D.R.; GUERRA JUNIOR, A.A.; CHERCHIGLIA, M.L.; ANDRADE, E.I.G. Judicialização do acesso a medicamentos no Estado de Minas Gerais, Brasil. Revista de Saúde Pública, São Paulo, v. 45, n. 3, jun. 2011.

MARIN, N.; LUIZA, V.L.; SERPA, C.G.; CASTRO, O.; SANTOS, O. M. Assistência Farmacêutica para gerentes municipais 2003. Disponível em: <http://www.opas. org.br/medicamentos/site/UploadArq/0080.pdfs. Acesso em: 05 out. 2011.

MARQUES, S.B. O princípio constitucional da integralidade de assistência à saúde e o projeto de lei n. 219/2007: interpretação e aplicabilidade pelo Poder Judiciário. Revista de Direito Sanitário, São Paulo, v. 10, n. 2, jul./out. 2009.

; DALLARI, S.G. Garantia do direito social à assistência farmacêutica no Estado de São Paulo. Revista de Saúde Pública, São Paulo, v. 41, n. 1, fev. 2007.

MINAS GERAIS. Portal da Transparência do Estado de Minas Gerais. Disponível em: <http://www.transparencia.mg.gov.br/>. Acesso em: 11 jan. 2012.

MINISTÉRIO DA SAÚDE. Secretaria de Atenção à Saúde/Departamento de Atenção Especializada/Coordenação Geral de Média e Alta Complexidade. Nota Técnica 2010: o acesso a medicamentos antineoplásicos no SUS. 2010. Disponível em: <http://portal.saude.gov.br/portal/arquivos/pdf/cgmac_ medicamentos.pdf>. Acesso em: 10 dez. 2011. 
PAULA, C. Silva. Uso off label de medicamentos: análise das demandas judiciais no CEMEPAR e conduta dos farmacêuticos no Paraná. 144 f. Dissertação (Mestrado em Ciências Farmacêuticas) - Universidade Federal do Paraná, Curitiba, 2010.

PEPE, V.L.E.; FIGUEIREDO, T.A.; SIMAS, L.; OSÓRIO-DE-CASTRO, S.G.C.; VENTURA, M. A judicialização da saúde e os novos desafios da gestão da assistência farmacêutica. Ciência \& Saúde Coletiva, Rio de Janeiro, v. 15, n. 5, ago. 2010.

PORTAL da Transparência do Estado de Minas Gerais. Disponível em: <http:// www.transparencia.mg.gov.br/despesas-do-estado?u=cHVibGljYWRvci5waHA ZGF0YT1ZV05oYjN4d2NtOTRhVzF2ZkhSbGJXRjhOSHhqYjJScFoyOTh OREV3TkRJNU1UY3dNaIF6TUh4MFpXMXdiMOpoYkdsa1IXUmxmRUZPVIV GTWZIVnVhV1JoWkdWOE1YeGhibTk4TWpBeE1 YeHVhWFpsYkh3MmZIU mxiR0Y4ZkhCaGFYdzBNVEEwTWpreE56QXIOQT09>. Acesso em: 11 jan. 2012.

ROCHE DO BRASIL. Bula dos produtos. Disponível em: <http://www.roche.com. $\mathrm{br} /$ portal/rochebrazil//ista_de_produtos?paf_dm=full\&categoryld=re7193008_ ct57043>. Acesso em: 10 maio 2011.

VIEIRA, F. S. Ações judiciais e direito à saúde: reflexão sobre a observância aos princípios do SUS. Revista de Saúde Pública, São Paulo, v. 42, n. 2, abr. 2008.

WANNMACHER, L. A ética do medicamento: múltiplos cenários. Ministério da Saúde. Uso racional de medicamentos: temas selecionados. Brasília, v. 4, n.8, jul. 2007. 